\title{
Desempleo y protección social: el caso colombiano*
}

\author{
Unemployment and social protection: The Colombian case
}

Desemprego e proteção social: o Tópico colombiano

\author{
Laura Daniela Londoño-Upegui1; Luz Mery Mejía-Ortega2 \\ 1 Administradora en Salud: énfasis en gestión de servicios de salud, Universidad de Antioquia, Medellín-Colombia. lauralup7@gmail. \\ com. ORCID: https://orcid.org/0000-0002-9418-8299 \\ 2 Magíster y doctora en Salud Pública. Universidad de Antioquia. mery.mejia@udea.edu.co.orcid: https://orcid.org/0000-0002- \\ 0917-3704
}

Recibido: 18/11/2018. Aprobado: 23/07/2019. Publicado: 01/10/2019

Londoño-Upegui LD, Mejía-Ortega LM. Desempleo y protección social: el caso colombiano. Rev. Fac. Nac. Salud Pública. 2019;37(3):54-63. DoI:10.17533/udea.rfnsp.v37n3a07

\section{Resumen}

El objetivo de este ensayo es reconocer el fenómeno del desempleo como un problema que afecta el bienestar social y que requiere, por ello, respuestas del Estado y la sociedad en materia de protección social. Asimismo, se discuten los mecanismos de protección social contra el desempleo en el caso colombiano.

-Palabras clave: desempleo, protección social, estado de bienestar, Colombia.

\section{Abstract}

The objective of this essay is to recognize the unemployment phenomenon as a problem that affects social welfare and requires, therefore, responses from the State and society in terms of social protection. Likewise, social protection mechanisms against unemployment in Colombia are also discussed.

---------Keywords: unemployment, social protection, welfare state, Colombia.

\section{Resumo}

O objetivo desta apostilha é reconhecer o fenômeno do desemprego como um problema que afeta o bem-estar social e que exige, portanto, respostas do Estado e da sociedade atinentes à proteção social. Da mesma forma, são discutidos mecanismos de proteção social contra o desemprego no Tópico colombiano.

---------Palavras-chave: desemprego, proteção social, estado assistencial, Colômbia

* El presente artículo se deriva del trabajo de grado "El seguro de desempleo como mecanismo de protección social: perspectiva histórica y situación actual en Colombia, 2017”, cuya autoría es de Laura Daniela Londoño Upegui. Con respecto a este artículo, las autoras agradecen a los profesores Emmanuel Nieto y Marcela López, profesores de la Facultad Nacional de Salud Pública, quienes como jurados del trabajo de grado hicieron recomendaciones para el mejoramiento del informe final, de donde se genera este artículo. 


\section{Introducción}

El desempleo es un fenómeno social que no solo concierne a la persona que carece de un trabajo que le proporcione una fuente formal y permanente de ingresos, sino que incide directamente sobre distintos ámbitos de la vida privada, familiar y social [1]. Este fenómeno afecta negativamente la satisfacción de expectativas y de necesidades humanas, en tanto menoscaba la vida cotidiana de quien lo sufre, su entorno familiar y social, su situación financiera, de vivienda y la salud $[2,3]$. Aunque muchos Estados y gobiernos en el mundo han desarrollado estrategias y acciones políticas para mitigar esta problemática, pareciera que dichos esfuerzos han sido insuficientes [4].

La Organización Internacional del Trabajo (OIT), en su más reciente informe, muestra que la tasa de desempleo a nivel mundial desde el año 2017 no ha presentado mayor variación, e incluso ha mostrado una tendencia a la baja, pasando del 5,1\% en 2017 al 4,9\% en 2019. Sin embargo, en cifras absolutas, el mismo informe muestra que la tendencia es hacia un incremento de un millón de personas desempleadas cada año, por lo cual se espera que en 2020 haya un total de 174 millones de personas sin empleo en el mundo [5].

El informe de la OIT, además, indica una tendencia distinta de la tasa de desempleo para los países según nivel de ingresos. Así, para los países de bajos ingresos, la tasa de desempleo se mantiene en 3,7\% desde 2017, y se proyecta sin variación hasta 2020. Para los países con medio-alto nivel de ingresos, esa tasa alcanza el $6,0 \%$, sin modificación entre 2017 y 2020 [5]. Para el caso de América Latina y el Caribe, esta tasa, en 2017, era de $8,1 \%$, y se espera que para 2020 se disminuya al $7,8 \%$ [5]. En Colombia, por su parte, la tasa de desempleo fue del $9,4 \%$ en 2017 y del $10,5 \%$ a mayo de 2019 , siendo todavía mayor para el conjunto de las 13 ciudades y áreas metropolitanas, donde la tasa alcanza el 11,2\% [6].

Conforme lo reconocen algunos autores, el desempleo está asociado a problemas estructurales socioeconómicos que requieren políticas integrales para su atención; entre ellas, las de protección social en caso de desempleo involuntario [7]. Según Gallie, Marsh y Vogler, el modo en que el desempleo afecta a quien lo padece depende básicamente de tres variables: las características de las instituciones de bienestar social que tienen que ver con el desempleo; los patrones de comportamiento familiar y social definidos por tradiciones culturales, y las condiciones específicas de cada sociedad, relativas al desarrollo y la estructuración de la economía [8].

En este mismo sentido, la oIT sustenta que el impacto de la crisis económica entre los grupos vulnerables es más alto en países con bajas coberturas de protección social, y que el $80 \%$ de la población mundial no tiene acceso a sistemas adecuados de salud y pensiones. En su "Informe mundial de seguridad social 2010-2011", indica que, en ese periodo, solo el $20 \%$ de la población en edad de trabajar y sus familias poseían acceso efectivo a las garantías plenas de protección social $[9,10]$.

$\mathrm{Al}$ respecto, Mejía [11], en su tesis doctoral, afirma que la precariedad en la garantía del cuidado de la calidad de vida y del bienestar de la población en el mundo actual, frecuentemente asociada a la condición de desempleo, a la tendencial caída de la calidad del empleo, y a los altos niveles de pobreza, se ha documentado en numerosos estudios, que dicen bien a las claras de la necesidad de la búsqueda de alternativas de protección social en situación de desempleo.

En tal sentido, se ha documentado que la desprotección social a causa de largos periodos de desempleo desencadena complejos problemas sociofamiliares, de salud mental, aumento del consumo de sustancias nocivas para la salud (como cigarrillo, alcohol y sustancias psicoactivas), así como significativos obstáculos para ejercer el derecho a la salud. De igual modo, esta situación desestructura el sentido y el significado de la vida cotidiana de quienes la sufren [11].

De acuerdo con la Comisión de los Determinantes Sociales de la Salud de la Organización Mundial de la Salud, todas las personas necesitan protección social a lo largo de la vida, y muy especialmente en circunstancias adversas de enfermedad, discapacidad, invalidez y desempleo involuntario [12]. Este último, entendido como el reflejo de decisiones políticas y económicas que se adoptan en cada sociedad, y reconocido como uno de los más fuertes condicionantes de la salud y causante de la precariedad del bienestar y de las condiciones de vida de la población [13]. En consecuencia, desde la teoría sociológica de Esping-Andersen, se postula la necesidad de que la sociedad y los gobiernos definan políticas públicas de protección social que intervengan la problemática del desempleo y sus efectos sociales [11].

Tal como lo reconoce la OIT [14], la protección social, como derecho humano —-según la Declaración Universal de los Derechos Humanos-, desempeña un papel decisivo en el logro del desarrollo sostenible, la justicia social y el derecho a la seguridad social para todos. Con base en esta aseveración, y para los propósitos de este artículo, las autoras compartimos el concepto de protección social enunciado por la oIT, entendido como

[...] un conjunto de políticas y programas diseñados para reducir y prevenir la pobreza y la vulnerabilidad en todo el ciclo de vida. Abarca los beneficios familiares y por niño, las prestaciones de maternidad, desempleo, accidentes del trabajo y enfermedades profesionales, así como las pensiones de vejez, invalidez 
y sobrevivientes, y la protección de la salud. Los sistemas de protección social abordan todas estas ramas mediante una combinación de regímenes contributivos (seguro social) y de prestaciones no contributivas financiadas con impuestos, en particular la asistencia social [14, p. 216].

Dicho lo anterior, el propósito de este ensayo es aportar sobre el desempleo y la protección social que, sin lugar a dudas, son de interés en el campo de la salud pública y la seguridad social, desde la temática de la protección contra el desempleo, sus repercusiones $\mathrm{y}$ la desprotección y los mecanismos de protección en Colombia, sustentados desde la comprensión sociológica del estado de bienestar, legislativa, histórica y de la protección social.

\section{Las repercusiones del desempleo y la desprotección social sobre el bienestar}

Como lo muestran distintos autores y organizaciones internacionales, en las últimas décadas los niveles de desempleo se han elevado en todo el mundo. Como lo afirma Goyeneche [3], el mercado laboral, en los últimos años, ha tenido la singularidad de su inestabilidad y el aumento del desempleo, lo que significa que los riesgos se elevan para las personas afectadas por el desempleo, acarreando nuevas problemáticas sociales y mayores niveles de pobreza. Mejía [12], soportada en otros autores, afirma que: "el desempleo y la desprotección social generan múltiples desequilibrios sociales que, a su vez, desencadenan mayor riesgo de que la población sufra condiciones de pobreza, que franjas adicionales se precipiten a ella, y que, consecuentemente, [los desempleados] permanezcan marginados de las estructuras sociales [...]" [12, p. 1].

Como lo expone Mejía, múltiples investigaciones han demostrado que entre la población desempleada existen mayores tasas de morbimortalidad general y de desnutrición; creciente tendencia a problemas de baja autoestima, depresión, alcoholismo, conflictos familiares; sentimientos de inseguridad, de fracaso y desesperanza; aumento de conductas suicidas y de expresiones de violencia, así como mayores gastos en salud por cuenta propia, y mayor riesgo de tabaquismo y de obesidad, entre otros problemas de salud pública [12].

En el marco de lo expresado anteriormente, Salvia y Pla [15] recalcan que la pérdida de trabajo implica el desgaste del desarrollo de las personas y una limitante para construir un sistema socioeconómico fundado en el bien común y con un panorama de bienestar para todas las personas. Especialmente, el desempleo acarrea las problemáticas en el sistema social en, por lo menos, tres sentidos:
1. Degrada la capacidad de trabajo establecida, afectando habilidades, destrezas y conocimientos previamente adquiridos por las personas con experiencia de empleo; 2. Devalúa el valor económico y simbólico del trabajo y afectan la legitimidad de las normas del derecho laboral en personas que recién ingresan al mundo del trabajo, y 3. Debilita la cultura del trabajo al desmotivar, frustrar y atemorizar a aquellos trabajadores y familias que experimentan una situación ocupacional desfavorable [15, p. 2].

Para Kliksberg [16], la pobreza y la problemática del desempleo confluyen en el aumento de los trabajos informales, y a su vez en la desprotección social. Para este autor, es claro que dicha situación conduce a un alto porcentaje de trabajos inestables, con carencias de los beneficios de la seguridad social y, por tanto, a mayores niveles de malestar y de desigualdad social. En el mismo sentido, Moyano et al. expresan que el desempleo afecta negativamente la satisfacción en varios ámbitos de la vida de las personas, como su actividad cotidianasocial, su situación financiera económica, su situación de vivienda, y la salud, siendo el tiempo de ocio el único que se ve favorecido [2].

Como lo reconoce Goyeneche, como el trabajo es estructurador de la vida cotidiana (provee una estructura horaria a la persona, vínculos, participación en proyectos colectivos, estatus social e identidad), con la pérdida del mismo se pierde todo lo que de él se deriva. Además de la pérdida del ingreso y de la afectación de su vida cotidiana, el desocupado sufre de pérdida de autoestima y motivación, vive frustración, resignación y se vuelve pasivo, con las respectivas consecuencias en su entorno familiar y social. Además, el autor también afirma que las personas desempleadas por largos periodos tienden a perder sus conocimientos y habilidades, debido a que no poseen oportunidades de practicar su trabajo u oficio, y de actualizarse, además de la notable disminución de sus ingresos [3].

Por consiguiente, según Padilla et al., la disminución de ingresos lleva al desempleado a la afectación de viviren un medio inseguro. Los recursos económicos componen la principal fuente de control instrumental económico que posee una persona en la sociedad. Los escenarios de pobreza se asocian a la desprotección, lo que puede conducir al desempleado al deterioro de su bienestar. Pero, sin duda, los apoyos económicos que la persona desempleada perciba y su disponibilidad de recursos mitigan los efectos negativos del desempleo [17].

Al respecto, Acosta y Ramírez recalcan que las personas con mayores ingresos tienen menor posibilidad de que las problemáticas de la crisis los afecten, ya que poseen mejores y mayores alternativas para contrarrestar una problemática de ingresos. Para la 
población que no goza de estas condiciones favorables por su parte, es necesario disponer de mecanismos de bienestar diferentes al salario, como lo son el acceso a la protección básica y a la posesión de activos para reducir la vulnerabilidad individual, familiar y social [18].

Sostienen también que la pérdida o falta de empleo, especialmente para algunos tipos poblacionales, como lo son las mujeres, los jóvenes y minorías étnicas; la disminución de ingresos a causa del desempleo de la persona encargada del sostenimiento familiar, y los efectos negativos sobre las personas, causados por el desempleo, la deserción escolar, la falta de atención en salud y deficiente nutrición son algunas de las problemáticas que constituyen el foco de la creación de los programas de protección [18].

\section{La protección contra el desempleo en el marco del estado de bienestar}

Para autores como Benavides, "el concepto de Estado del Bienestar está asociado con la capacidad de una sociedad para satisfacer necesidades básicas de las personas que la componen, como la educación, el trabajo o la salud, entre otras" [19, p. 1]. Para otros, como Moreno, el estado de bienestar se define como el conjunto de entes públicos que brindan servicios sociales que buscan mejorar las condiciones de vida y promover la igualdad de oportunidades de las personas [20].

Para Briceño y Gillezeau, el estado de bienestar alude a lo que comúnmente se conoce como satisfacción y provisión de algunas necesidades básicas como las de salud, económicas y educativas, brindadas desde entes públicos y privados, con la intervención del Estado como ente regulador y garante [21]. Para estos autores, el inicio de esta intervención estatal se remonta al siglo XIX en Alemania, donde Bismarck diseñó una legislación social sobre la ley del seguro de enfermedad, con el cual se dieron los primeros programas estatales de previsión social. Igualmente, con este se establecieron las bases de la seguridad social moderna bajo el principio contributivo (que alude a que la población debe realizar contribuciones, en la medida de sus posibilidades económicas, para el financiamiento y el sostenimiento de la seguridad social), mediante el cual se financiaba un sistema básico de previsión social [21].

Castell y Bosh afirman que "la comprensión del Estado de Bienestar se hace difícil con la presencia de altas tasas de desempleo, debido precisamente a que el Estado tiene como objeto la sostenibilidad del mercado laboral" [citados en 22, p. 132]. Para Salazar, el objetivo del estado de bienestar reside en mitigar las situaciones poco favorables, como la pobreza de las personas, que pueden terminar la cohesión social del sistema, instando al riesgo de la creación de valor económico. Las reformas sociales de amortiguación de la pobreza y brindar beneficios mínimos han sido referentes del estado de bienestar clásico. Según Salazar, en la actualidad, con los diferentes momentos de transformaciones de la organización económica mundial, se está recrudeciendo la marginalidad y la pobreza, no encontrándose soluciones políticas por parte de los diferentes Estados, poniendo así en riesgo la estabilidad del sistema [22].

Para Esping-Andersen [23], el estado del bienestar es la principal institución que permitió la construcción de diferentes modelos del capitalismo de la posguerra para la mejora social, aunque admite que no todos los estados del bienestar se han desarrollado del mismo modo. Para este autor, existen tres modelos o regímenes del estado del bienestar altamente diferenciados entre sí, organizados según su propia lógica y sus antecedentes históricos, y con formas de estratificación e integración social diferentes. Por tanto, también son distintas las maneras como se materializa la política social en cada uno de ellos. Esos modelos-regímenes del estado del bienestar los denomina de la siguiente manera: el conservador, el liberal y el socialdemócrata. Al respecto, advierte que cada uno de ellos adopta de forma distinta las políticas sociales en las que se reconocen y materializan ciertos derechos, prestaciones económicas y oportunidades para el bienestar social [23].

De acuerdo con este autor, el modelo conservador del estado de bienestar se caracteriza principalmente por la desmercantilización del trabajo, por lo cual supone que el bienestar social y la subsistencia no deberían depender del trabajo y de la productividad del empleo. Por ello, su principal punto de vista, o ideología, es que "la mercantilización de los individuos es moralmente degradante, socialmente corruptora, atomizadora y anómica" [23, p. 61]. Igualmente, el autor se refiere al modelo liberal, desde el cual el Estado solo interviene en aquellos asuntos y ámbitos en los que falla el mercado, y que, por tanto, solo debe asistir con subsidios a las personas que demuestren su real necesidad y su incapacidad de resolver sus necesidades en el mercado. De esta forma, se evita la extensión de los derechos sociales incondicionales y la "generosidad" del Gobierno se limita a los verdaderamente necesitados. Así, el modelo no induciría a los trabajadores a escoger la protección social por parte del Estado en lugar del trabajo. Por el contrario, se convertiría en un sistema de comprobación para los que no tienen medios de subsistencia y, por tanto, son incapaces de participar en el mercado [23].

En contraposición a este modelo de mercantilización de la fuerza del trabajo y de la seguridad social, se destaca el tercer modelo de bienestar: el socialdemócrata. Este es entendido por el autor como el mayor defensor del estado de bienestar en su más amplia dimensión. Este modelo asume que para lograr el mayor bienestar y 
la protección social universal es necesario ayudar a los verdaderamente necesitados. Por tal razón, se fundamenta en la solidaridad y en la defensa de los derechos sociales básicos, propios de la política social universalista [23]. En este modelo, las prestaciones sociales para el bienestar se fundamentan en las necesidades humanas y en los derechos sociales, y no en el trabajo o en el salario como factor determinante de las condiciones de vida de la población.

Según Esping-Andersen, ninguno de los tres tipos de regímenes defienden el pleno empleo como una parte integral de su compromiso de bienestar; también expone que ningún modelo es totalmente puro, ya que entre los criterios esenciales para definir los estados de bienestar tienen que ver con la calidad de los derechos sociales, la estratificación social y la relación existente entre el Estado, el mercado y la familia, ya que obviamente el mundo está compuesto por distintos grupos de regímenes [23].

En correspondencia con los planteamientos de Esping-Andersen, Navarro afirma que, cuando se habla de política social y de regímenes del bienestar, se alude principalmente a la administración pública de los riesgos intrínsecos a las relaciones sociales, en las que básicamente el trabajo está mercantilizado. Según este autor, la dependencia de las personas de los ingresos laborales constituye el principal factor de riesgo en las sociedades capitalistas, ya que la ausencia de esos ingresos afecta la supervivencia de aquellas [24].

Como lo explica Navarro, Esping-Andersen describe el enfoque de los modelos del bienestar y crea una "tipología", que se basa principalmente en el análisis de algunas áreas, como lo son las relaciones entre Estado y mercado, la estratificación, y la desmercantilización. Estas configuraciones de los regímenes de bienestar resultantes constituyen grupos o asociaciones en los que las instituciones se conjugan para crear modelos de gestión pública de riesgos, lo que implica, a su vez, diferentes modelos de solidaridad social, que integran las relaciones entre el Estado, el mercado de trabajo, la familia y el sistema de estratificación social [24].

Al respecto, Del Pinto, citando a Esping-Andersen, concluye que los derechos sociales pocas veces son incondicionales, y en el caso de quienes reciben subsidios, los solicitantes al menos tienen que cumplir situaciones poco favorables, como la enfermedad, la pobreza o el desempleo. Sin embargo, además de la sola presencia de un problema, las condiciones suelen estar ligadas al tipo de convenio con la seguridad social [25]. Por su parte, para Savaris [26], el reconocimiento de los derechos económicos, sociales y culturales (DESC), en su conjunto, para todas las personas, implica la afirmación de su humanidad y su dignidad como un fin en sí mismo. Sin duda, la garantía de políticas públicas destinadas a hacer frente a las necesidades básicas de los individuos y sus familias constituye una base muy sólida para lograr una existencia digna, un proyecto de vida decente, el libre desarrollo de la personalidad, la cohesión y la integración social [26].

\section{El caso colombiano: los mecanismos de protección social contra el desempleo}

Como ya se ha indicado, la protección social contra el desempleo incluye distintos mecanismos, que van desde el ahorro individual, los seguros privados, la asistencia pública, los seguros de desempleo y las prestaciones sociales y económicas, legales y extralegales, todos ellos con distinto alcance y niveles de prestación, según la legislación de cada país [18].

Para el caso colombiano, la legislación laboral contempla dos tipos de prestaciones sociales económicas de protección contra el desempleo: las indemnizaciones, en caso de despido o terminación del contrato sin justa causa, y el auxilio de cesantías para los contratos laborales a término fijo e indefinido. Asimismo, dispone de un mecanismo de protección al cesante, el cual fue constituido inicialmente con la figura de "subsidio al desempleo" en el marco del sistema de protección social establecido mediante la Ley 789 de 2002. De igual modo, dispone de una oferta de seguros contra el desempleo, ofrecidos por el mercado financiero del país. A continuación presentamos cada una de esas modalidades de protección social contra el desempleo.

\section{Las indemnizaciones y el auxilio de cesantías}

En el marco normativo del reconocimiento de derechos laborales en Colombia, en los inicios del año 1945 se expidió la Ley 6 de 1945 [27], donde se estipula que los empleadores quedan a cargo de las indemnizaciones por despido sin justa causa y las prestaciones de cesantías por despido o retiro voluntario del trabajador, entre otras ya legalmente establecidas. Mediante la Ley 90 de 1946 [28] se da origen al seguro social obligatorio y se crea la aplicación de este a los contratos laborales, adicional a las prestaciones de cobertura en salud por enfermedad no profesional y maternidad, accidentes de trabajo y enfermedades profesionales, y muerte. No obstante, en esta normatividad no se reconocieron prestaciones de protección al desempleo, los cuales existían en otros países desde décadas anteriores [29].

En este contexto, uno de los referentes normativos más importantes de esta materia, que ratificaron las disposiciones previas y buscaron mayor justicia en las relaciones entre empleadores y trabajadores, es el Código Sustantivo del Trabajo, adoptado el 5 de agosto de 1950, mediante el Decreto Ley 2663 [30]. Esta norma 
estipula que, en caso de terminación sin justa causa del contrato de trabajo, el empleador debe garantizar una indemnización económica al trabajador, proporcional al tiempo laborado y último salario devengado.

Según el artículo 64 del Código Sustantivo de Trabajo, esta indemnización económica para el trabajador se liquida de manera distinta según sea el tipo de contrato de trabajo que se termina sin justa causa: a término indefinido, a término fijo, por duración de obra o labor, y ocasional o transitorio. Para el caso del contrato de trabajo a término indefinido, la indemnización también se liquida de manera diferencial, según fuera el salario del trabajador al momento de su despido. Estas bases de liquidación se estipulan así: salarios inferiores a 10 salarios mínimos mensuales legales vigentes (smmlv), salarios iguales o superiores a los $10 \mathrm{smmlv}$, y vinculaciones inferiores a un año [30]. Para el caso del desempleado por justa causa, no se le paga ninguna indemnización.

Cabe anotar que, debido a las políticas de flexibilización laboral que se llevan a cabo en Colombia con gran auge desde la expedición de la Ley 50 de 1990, cada vez es menor la proporción de trabajadores que tienen derecho a esta prestación económica de protección contra el desempleo. Tal situación se presenta porque, desde entonces y hasta la fecha, son más frecuentes los contratos de trabajo a término fijo inferiores a un año o por prestación de servicios, ante los cuales no se garantiza esta prestación [31]. Similar pérdida se da con las prestaciones sociales de cesantía, a las cuales nos referimos a continuación.

En cuanto a las cesantías, esta prestación económica, a cargo del empleador, en principio se otorgó solamente a los trabajadores del sector público [27], y más tarde, a partir de la expedición del Decreto 2663 de 1950, se extendió a los del sector privado. Tal prestación se estipuló como un auxilio a los trabajadores, con el fin de cubrir sus necesidades económicas durante el periodo de cesantía laboral, constituyéndose así en un ahorro obligado destinado a cubrir el riesgo de desempleo.

Según el artículo 253 del Código Sustantivo del Trabajo, la base para liquidar el auxilio de cesantías es distinta según sea el tipo de salario del trabajador. Si el salario es fijo sin variación en los últimos tres meses, se toma este valor como base de liquidación. Si ha tenido variación en los últimos tres meses, se toma como base el promedio del último año, o de todo el tiempo servido si fuera menor a un año. Para determinar la base de liquidación, la norma establece que se deben tener en cuenta todos los conceptos que constituyen salario, como: el sueldo, las comisiones, el auxilio de transporte, las horas extras y los recargos nocturnos, dominicales y festivos. El monto de esta prestación, según el artículo 249 del mismo código, corresponde a un salario por cada año laborado, o proporcional, si el tiempo fuese inferior a un año.

Al respecto, Romero y Cortés [32] indican que, para 1950, las cesantías contaban con mayor relevancia económica de la que tienen en la actualidad, por su forma de liquidación retroactiva, lo cual se constituía en una fuente efectiva de protección económica durante el desempleo. Sin embargo, la Ley 50 de 1990 y demás normas posteriores hicieron que, en gran medida, se redujera ese poder adquisitivo para los trabajadores cesantes, en tanto las cesantías ya no son retroactivas, sino que son liquidadas anualmente y transferidas por el empleador a los Fondos de Cesantías [32]. Según la norma, una vez transferidas, el trabajador puede disponer de ellas a modo de retiro parcial o total con fines de adquisición, construcción o mejoras de la vivienda, o de educación propia, del cónyuge o de los hijos.

$\mathrm{Al}$ respecto, Bardey, Kiuhan y Suárez exponen que, en Colombia, los requisitos para retirar las cesantías son poco restrictivos, y que esto posibilita la destinación a otros fines distintos a los ya permitidos por la norma, al tiempo que pueden perder la finalidad de socorrer al trabajador y su familia durante la contingencia del desempleo [33].

\section{El subsidio al desempleo y el actual mecanismo de protección al cesante}

En Colombia, el tema de los subsidios para el desempleo se trató por primera vez con la expedición de la Ley 789 del año 2002 [34], mediante la cual se regularon aspectos normativos de apoyo al empleo, de ampliación de la protección social y modificación de algunos artículos del Código Sustantivo del Trabajo. En esta ley se definió el sistema de protección social como "el conjunto de políticas públicas orientadas a disminuir la vulnerabilidad y a mejorar la calidad de vida de los colombianos, especialmente de los más desprotegidos. Para obtener como mínimo el derecho a: la salud, la pensión y al trabajo" [34, p. 1].

Asimismo, mediante esta ley se creó el Fondo de Protección Social, cuyo objeto principal sería la financiación de programas sociales para el subsidio al empleo para la pequeña y la mediana empresa, y la definición de recursos para el fomento del empleo y la protección durante el desempleo, a través de las cajas de compensación familiar. En lo correspondiente al régimen de protección al desempleado, esta ley creó el Fondo de Subsidio al Empleo y al Desempleo como una cuenta especial adscrita al Ministerio de Trabajo y Seguridad Social, sin personería jurídica y cuyos recursos eran administrados mediante fiducia pública [34].

De este modo, se creó en Colombia el primer subsidio al desempleo, el cual consistía en un 1,5 smmlv, distribuido en seis cuotas iguales, no otorgadas en dinero, 
sino en bonos para alimentación, educación o pago de la seguridad social. Los beneficiarios de este subsidio solo podrían recibirlo una sola vez en su vida, y para ello debían cumplir con los requisitos establecidos por el Gobierno nacional, como el requerimiento de que la persona hubiese realizado aportes durante un año (o dos para trabajadores independientes) a una caja de compensación familiar y demostrar que estaba desempleado [34].

Dicho subsidio de desempleo fue sustituido por lo que hoy se conoce como el mecanismo de protección al cesante, denominado por muchos como el "seguro de seguro de desempleo colombiano", creado mediante la Ley 1636 de junio de 2013 [35], bajo la administración de las cajas de compensación familiar. Este fondo tiene como objetivo garantizar la protección social de los trabajadores en caso de quedar desempleados y que cumplan los requisitos para ser beneficiarios. $\mathrm{Su}$ valor equivale a 1,5 smmlv y es otorgado en seis mensualidades iguales, de los cuales se descuenta la cotización al sistema de salud y pensiones. El resto se entrega como cuota monetaria de subsidio familiar y en bonos de alimentación. Adicionalmente, ofrece los servicios de capacitación para el empleo.

El Ministerio del Trabajo, en su "Informe de rendición de cuentas" del año 2014, expone que desde el inicio de la puesta en marcha del mecanismo de protección al cesante se recibieron, para ese año, aproximadamente 13844 solicitudes para acceder a los beneficios que otorga el mismo, de las cuales se aceptaron más de 10000 postulaciones de personas cesantes. El resultado de esto fue que 8600 colombianos recibieron los beneficios del seguro de desempleo ese año [36].

En su más reciente informe al Congreso del periodo 2016-2017, el Ministerio informa que entre 2014 y 2017 - el tiempo en que se desarrolló este mecanismo de protección social-, a nivel nacional se asignaron beneficios de prestaciones económicas a 428704 personas desempleadas, y se capacitaron 678829 personas en condición de desempleo. Asimismo, en ese periodo, se colocaron laboralmente 368372 personas, equivalentes al 8,2\% del total de 3035671 hojas de vida registradas en la Agencia Pública de Empleo [37]. En este informe bien puede apreciarse el importante incremento, cada año, de los beneficiarios de las prestaciones económicas y de capacitación que ofrece este mecanismo. Al tiempo, se evidencia un importante aumento del registro de personas desempleadas solicitantes de este beneficio, así: en 2014, un total de 345073 personas registradas; en 2015, fueron 773809 ; en 2016, 864 529; y en 2017, el total fue de 1052060 personas aspirantes a los beneficios del mecanismo de protección al cesante.

\section{Los seguros privados de desempleo}

En Colombia, las compañías de seguros y las entidades del sector financiero están facultadas por la Superintendencia Financiera para ofrecer el seguro privado de desempleo. En general, las entidades bancarias son las poseen mayor interés en que las personas, clientas de sus servicios de crédito, adquieran dichos seguros, con el fin de garantizar el cumplimiento de la obligación financiera en caso de desempleo [38,39].

De acuerdo con Zúñiga, muchas de las compañías que ofrecen estos seguros de desempleo en Colombia no ofrecen este seguro como un contrato principal privado, sino como un contrato adherido a sus productos y servicios ofrecidos en general, o en relación con los contratos que adquiera el usuario de estas compañías. En efecto, muchas de estas compañías ofrecen algunos de sus servicios de crédito financiero condicionado a la adquisición del seguro de desempleo, caso en el cual deja de ser un seguro voluntario [40].

Además, como lo exponen Bardey, Kiuhan y Suárez [33], las personas que deciden acceder a este tipo de seguro de manera voluntaria o como requisito de un servicio de crédito financiero, deben demostrar un contrato de trabajo a término indefinido, por obra o labor, de asociación, con plazo presuntivo, y los demás que contemple la legislación laboral. Según los autores, cada entidad define el valor del seguro y los amparos del contrato de aseguramiento. Entre esos amparos, por lo general se cubre la pérdida de empleo por renuncia, por terminación del contrato por cualquier modalidad sin justa causa, declaración de insubsistencia, mutuo acuerdo o terminación anticipada del contrato, pero en ningún caso por el despido con justa causa.

\section{Discusión y conclusiones}

Para comprender la necesidad de la protección social durante el desempleo, es preciso reconocer que el trabajo, como fuente principal de ingresos para la subsistencia humana, es estructurador de la vida cotidiana, la identidad, el estatus y la pertenencia social, en tanto provee los recursos para vivir la vida, posibilita la organización social y la construcción de vínculos sociales para la participación e inclusión en los proyectos colectivos [2]. Lo anterior permite entender que el desempleo, como fenómeno social, no afecta solo al sujeto que lo sufre, sino también a su grupo familiar y su entorno social [2,3]. En tal sentido, como lo afirma Rodríguez [41], el Estado puede asumir la responsabilidad de afrontar dicho fenómeno no solo actuando como intermediador laboral, sino también como garante de los programas 
de protección laboral y de asistencia social para aquellos en situación de desempleo.

Como lo expone Mejía [11], ante la dificultad de las sociedades actuales para garantizar el pleno empleo, se requieren respuestas políticas y sociales que, en la perspectiva de construir sociedades justas, equitativas, democráticas e incluyentes, les permitan a las personas desempleadas y sus familias hacer frente a los eventos de la vida cotidiana durante el tiempo de desocupación [11]. Asimismo, como lo destaca Cortés [42], la protección social es la respuesta a las situaciones de problemáticas individuales y sociales que deben ser remediadas colectivamente, ya que estas no solo pueden dejarse a la gestión e iniciativas individuales, ya sea por la incapacidad de su manejo de manera individual, como por los bienes y esfuerzos que comprometen la superación de estos. Este planteamiento se asumió como postura en este trabajo, pues las autoras comprenden que los asuntos de la protección social no son de responsabilidad exclusiva de cada persona, sino de responsabilidad compartida entre el Estado, el individuo y la familia, cuando estos últimos dispongan de los recursos económicos para esa protección, y de responsabilidad exclusiva del Estado, cuando así se requiera.

En este sentido, Franco y Mejía exponen la necesidad de que se implementen sistemas de protección social adecuados, en consideración de que estos, a su vez, están condicionados por sus diferentes modelos de desarrollo y por las constantes reformas estructurales (económicas y sociales) que se han hecho a los Estados [43].

En materia de empleo y protección social en Colombia, la Ley 50 de 1990 sin duda representó un hito de ruptura de las garantías del contrato de trabajo a término indefinido, y de las garantías laborales y de seguridad social que se derivaban del mismo. La más notable consecuencia de ese quiebre en el mercado laboral colombiano, desde entonces, es la flexibilidad laboral, que a su vez se expresa en precariedad laboral, informalidad del empleo, desempleo y desprotección social. Todo ello, no obstante los insuficientes intentos gubernamentales por remediar la situación mediante el mecanismo de protección al cesante que se lleva a cabo desde el año 2014, posterior a la también insuficiente modalidad de subsidio al desempleo creado en 2002. A todas luces, dicha flexibilidad laboral, producto a su vez de las políticas de reforma estructural y neoliberal del Estado, va en contravía de los DESC que promueve el estado de bienestar, entre los cuales se destacan el derecho al trabajo digno y a la seguridad y la protección social.

Al respecto, Núñez y Cuesta destacan que, a pesar de los esfuerzos realizados en las últimas décadas en Colombia, todavía no se crea un sistema de protección social que desempeñe totalmente sus funciones principales de brindar asistencia social a quienes lo requieren y asegurar a la población contra los cambios y la crisis en la economía. Según los autores, pese a que existen mecanismos para cubrir algunos riesgos, gran parte de la población trabajadora informal no se encuentra protegida contra los riesgos de enfermedad, vejez, invalidez o desempleo [44].

De igual manera, Velásquez [45] señala que es preciso reconocer que una de las grandes problemáticas del mercado laboral colombiano es la falta de protección efectiva ante el desempleo. Tal es el caso en lo evidenciado en este estudio, en donde las indemnizaciones por despido no aseguran los ingresos en los periodos de desempleo. En el caso de las cesantías, estas se utilizan en gran parte para los fines de consumo estipulados por la ley, lo que disminuye su suficiencia $\mathrm{y}$ alcance. $\mathrm{Y}$ en el caso del mecanismo de protección al cesante, este no asegura una provisión significativa de beneficios monetarios que suplan la pérdida del salario de las personas que quedaron sin empleo, y por ello las personas recurren a sus propios fondos de cesantías para afrontar la crisis [45].

En este sentido, Núñez y Cuesta [44] refieren que el sistema de protección social colombiano no está cumpliendo con su objetivo principal, ya que sus programas cuentan con una baja e ineficiente cobertura, es ineficiente en la focalización, posee altos costos administrativos y sus posibilidades de tener economías en escala son bajas. Los autores expresan que la coordinación entre las entidades y los niveles gubernamentales son deficientes, y que en el esquema actual no existe un mecanismo bien definido para la protección de los más pobres ante efectos de choques covariados, que afectan simultáneamente a un grupo de individuos o familias.

Por lo anterior, es posible concluir que ante la evidencia de los efectos negativos del desempleo sobre la calidad de vida y el bienestar general de quienes lo sufren, el Estado debería garantizar las políticas y estrategias necesarias para lograr óptimas condiciones de protección social, especialmente para las personas en situación de desempleo, conforme a los postulados del estado de bienestar y de los DESC. En Colombia se destacan algunos avances en materia de protección social contra el desempleo. No obstante, ante la creciente situación de desempleo y precariedad laboral, esos avances todavía son insuficientes para garantizar condiciones básicas de bienestar y protección social. Por tanto, mayores recursos y estrategias de protección más integrales y universales son fundamentales de implementarse. 


\section{Conflicto de interés}

Las autoras declaran no tener conflictos de interés.

\section{Declaración de responsabilidad}

Se declara que los puntos de vista expresados son responsabilidad de las autoras.

\section{Referencias}

1. Aparicio Cabrera A. Efectos psicosociales del desempleo. Rev Investig Soc [internet]. 2006 [citado 2017 may. 2]; 2(3):6782. Disponible en: http://biblat.unam.mx/es/revista/revistade-investigacion-social/articulo/efectos-psicosociales-deldesempleo.

2 Moyano Díaz E, Gutierrez Peña D, Zúñiga Cabrera $\mathrm{K}$, et al. Empleados y desempleados, atribuciones causales y bienestar subjetivo. Psicol Soc [internet]. 2013 [citado 2017 may. 9]; 25(2):440-50. Disponible en: http://www.scielo.br/pdf/psoc/ v25n2/21.pdf.

3. Goyeneche AP. Un análisis de las políticas de Estado frente al desempleo. En: Sociedade Brasileira de Sociologia, XII Congresso Brasileiro de Sociología, Belo Horizonte-MG, 31 de maio a 03 de junho; 2005. pp. 1-8.

4. Yánez Contreras M, Cano Hernández K. Tipología del desempleo: un análisis a través de la Curva de Beveridge. 2011 [citado 2017 may. 2]; (35):215-233. Disponible en: http://www.redalyc.org/ pdf/1942/194224362012.pdf.

5 International Labour Office (ILO). World Employment and Social Outlook. Trends 2019. Geneva: International Labour Office [internet]; 2019 [citado 2019 jun. 29]. Disponible en: https:// www.ilo.org/wcmsp5/groups/public/---dgreports/---dcomm/--publ/documents/publication/wcms_670542.pdf.

6. Departamento Administrativo Nacional de Estadística. Gran encuesta integrada de hogares (GEIH). Mercado laboral [internet]. 2019 [citado 2019 jun. 29]. Disponible en: https://www.dane.gov. co/index.php/estadisticas-por-tema/mercado-laboral/empleo-ydesempleo\#2017.

7. Ochoa León SM. Reporte temático núm. 3. El seguro de desempleo en México y el mundo [internet]. México: Cámara de Diputados, Centro de Estudios Sociales y de Opinión [citado 2017 jul. 27]; 2005. Disponible en: http://www3.diputados.gob.mx/camara/ content/download/21217/105397/file/ITSSS001\%20Seguro\%20 de\%20desempleo\%20en\%20Mexico....pdf.

8. Gallie D, Marsh C, Vogler CM. Social Change and the Experience of Unemployment [internet]. Oxford: Oxford University Press; 1994.

9. International Labour Organization (ILO). Employment and Social Protection Policies from Crisis to Recovery and Beyond: A Review of Experience. An ILO report to the G20 Labour and Employment Ministers Meeting. Washington, 20-21 april. Geneve: International Labour Organization [internet]; 2010 [citado 2017 jul. 27]. pp. 4-25. Disponible en: http://www.socialprotection.org/gimi/gess/RessourcePDF.action;jsessionid=qT2 LjerBjdebyLm4xLUZeAxkj4hpzbLGgzq-d5G8erYZubtq3k7t!348558363? ressource.ressourceId=23022.

10. International Labour Organization. World Social Security Report 2010/11. Providing coverage in times of crisis and beyond; 2010.

11. Mejía Ortega LM, A. Protección social en población desempleada 1997-2013: Un análisis en el contexto sociopolítico colombiano y en la voz de quienes sufren el desempleo en Medellín-Colombia [tesis doctoral]. [Medellín]: Universidad de Antioquia; 2015.

12. Mejía Ortega LM. Protección social de los desempleados del área urbana de Colombia: un asunto de interés para la investigación en salud pública. Rev Fac Nac Salud Pública [internet]. 2012 [citado 2017 may. 14]; 30:9-11. Disponible en: http://www.redalyc.org/ pdf/120/12025811025.pdf.

13. Wilkinson R, Marmot M. Los determinates sociales de la salud: Los hechos irrefutables [internet]. 2003 [citado 2017 jul. 28]; 1-29. Disponible en: http://www.bvsde.paho.org/bvsacd/ cd90/1008WILdet.pdf.

14. Organización Internacional del Trabajo. Informe mundial sobre la protección social. La protección social universal para alcanzar los Objetivos de Desarrollo Sostenible. 2017-2019. Ginebra [internet]; 2017 [citado 2019 jun. 29]. Disponible en: https:// www.ilo.org/wcmsp5/groups/public/---dgreports/---dcomm/ documents/publication/wcms_624890.pdf.

15. Salvia A, Plá J. El otro desempleo. Impacto del crecimiento sobre la estructura del empleo durante los últimos cuatro años. La Causa Laboral [internet]. 2009 [citado 2017 jul. 6]; (38):1-15. Disponible en: https://www.aacademica.org/agustin.salvia/55.pdf.

16. Kliksberg B. América Latina: una región en riesgo. Pobreza, inequidad e institucionalidad social. Economía y Sociedad [internet]. 2000 [citado 2017 jul. 6]; 5(14):5-33. Disponible en: http://www.revistas.una.ac.cr/index.php/economia/article/ view/1539/1458.

17. Martín Padilla E, Fajardo Gonzales C, Gutiérrez Morales A, Palma Prieto D. Estrategias de afrontamiento de crisis causadas por desempleo en familias con hijos adolescentes en Bogotá. Act. Colom. Psicol. [internet]. 2007 [citado 2017 may. 14]; 10(2):127-41. Disponible en: http://www.scielo.org.co/scielo. php?script=sci_arttext\&pid=S0123-91552007000200013.

18. Acosta OL, Ramírez JC. Las redes de protección social: modelo incompleto. Santiago de Chile: CEPAL [internet]; 2004 [citado 2017 jul. 6]. Disponible en: http://repositorio.cepal.org/bitstream/ handle/11362/5124/S04142_es.pdf?sequence=1\&isAllowed=y.

19. Benavides FG. Salud pública y seguridad social, dos componentes básicos del estado del bienestar. Gac Sanit [internet]. 2011 [citado 2017 may. 8]; 25(2):91-93. Disponible en: http://scielo.isciii.es/ pdf/gs/v25n2/editorial.pdf.

20. Moreno Fernández L (Ed.). Pobreza y exclusión: la "malla de seguridad" en España [internet]. Madrid: Consejo Superior de Investigaciones Científicas, Instituto de Estudios Sociales Avanzados [citado 2017 dic. 5]; 2001. Disponible en: https:// dialnet.unirioja.es/servlet/libro?codigo $=274703$.

21. Briceño W, Gillezeau P. Argumentos sobre el estado de bienestar. Negotium. 2012;(23):26-66.

22. Salazar Silva F. Teoría económica y Estado del Bienestar. Una aproximación. Cuad Adm [internet]. 2006 [citado 2017 may. 8]; (35):127-43. Disponible en: http://www.redalyc.org/ pdf/2250/225020344006.pdf.

23. Esping-Andersen G. Los tres mundos del estado del bienestar. Madrid: Edicions Alfons El Magnànim, 1993; 1993.

24. Navarro Ruvalcaba MA. Modelos y regímenes de bienestar social en una perspectiva comparativa: Europa, Estados Unidos y América Latina. Desacatos [internet]. 2006 [citado 2017 jun. 20]; (21):109-34. Disponible en: http://www.redalyc.org/ pdf/139/13902108.pdf.

25. Del Pinto E. Seis debates sobre los Estados de Bienestar contemporáneos: capacidad de reforma, resultados, nuevos riesgos, globalización, sostenibilidad y alternativas de futuro [internet]; 2011 [citado 2017 jun. 20]. pp. 1-16. Disponible en: 
http://americo.usal.es/iberoame/sites/default/files/del_pino_ debates_estados_bienestar.pdf.

26. Savaris JA. Globalización, crisis económica, consecuencialismo y la aplicación de los derechos económicos, sociales y culturales (DESC). Prolegómenos. Derechos y Valores [internet]. 2012 [citado 2017 jun. 29]; 15(30):21-44. Disponible en: http://www.redalyc. org/pdf/876/87625443002.pdf.

27. Colombia, Congreso de Colombia. Ley 6, por la cual se dictan algunas disposiciones sobre convenciones de trabajo, asociaciones profesionales, conflictos colectivos y jurisdicción especial de trabajo (1945 feb. 19)

28. Colombia, Congreso de Colombia. Ley 90, por la cual se establece el seguro social obligatorio y se crea el Instituto Colombiano de Seguros Sociales (1946 dic. 26).

29. Reina Salgado M. Mecanismo de protección al desempleado en Colombia : ¿en el camino de la protección laboral? [internet]. 2013 abr. [citado 2018 mar. 7]. Disponible en: http://library.fes. de/pdf-files/bueros/kolumbien/09894.pdf.

30. Colombia. Ministerio de protección social. Decreto 2663, Código sustantivo de trabajo (1950 ago. 5).

31. Gómez Vélez M. Sobre la flexibilidad laboral en Colombia y la precarización del empleo. Divers Perspect Psicol [internet]. 2014 [citado 2019 jun. 29]; 10(1):103-16. Disponible en: http://www. scielo.org.co/pdf/dpp/v10n1/v10n1a08.pdf.

32. Romero C, Cortés J. Las cesantías bajo la nueva normatividad. Rev Actual Labor [internet]. 2014 [citado 2018 mar. 5]; 183:11-6. Disponible en: http://legal.legis. com.co/document/Index?obra=rlaboral\&document=rlaboral fb691d433e600230e0430a0101510230.

33. Bardey D, Kiuhan S, Suárez J. Seguros de desempleo: revisión de literatura y propuesta para Colombia [internet]. 2009 jul. [citado 2017 ago. 10]. Disponible en: http://repository.urosario.edu.co/ bitstream/handle/10336/10885/5739.pdf.

34. Colombia, Congreso de Colombia. Ley 789, por la cual se dictan normas para apoyar el empleo y ampliar la protección social y se modifican algunos artículos del Código Sustantivo de Trabajo (2002 dic. 27)

35. Colombia, Congreso de la República. Ley 1636, por medio de la cual se crea el mecanismo de protección al cesante en Colombia (2013 jun. 18).

36. Colombia, Ministerio de Trabajo. Informe de Rendición de Cuentas [internet]; 2014 [citado 2018 mar. 7]. Disponible en: http://www.mintrabajo.gov.co/el-ministerio/control-yrendicion-de-cuentas/rendicion-de-cuentas/-/document_library/ DsTkNJMHfxZ9/view_file/265744.

37. Colombia, Ministerio del Trabajo. Informe de actividades sector trabajo al Congreso de la República 2016-2017 [internet]; 2017 [citado 2018 mar. 7]. Disponible en: http://www.mintrabajo. gov.co/web/guest/atencion-al-ciudadano/transparencia/informeenviado-al-congreso-de-la-republica/-/document_library/ FFmcDsnPm9MO/view_file/58883883?_com_liferay_ document_library_web_portlet_DLPortlet_INSTANCE_ FFmcDsnPm9MO_redirect=ht.

38. La Crónica del Quindío. La Superintendencia Financiera entregó un concepto favorable respecto a la posibilidad de que las aseguradoras puedan comercializar seguros de desempleo [internet]. 2010 oct. 18 [citado 2018 mar. 7]. Disponible en: http://www.cronicadelquindio.com/noticia-completa-titulo-la superintendencia_financiera_entrego_un_concepto_favorable_ respecto_a_la_posibilidad_de_que_las_aseguradoras_puedan comercializar_seguros_de_desempleo-seccion-economicasnota-20200.htm.

39. Actualícese. ¿Qué tan beneficioso resulta aceptar los seguros de desempleo que ofrecen las entidades bancarias? [internet]. 2018 feb. 15 [citado 2018 mar. 27]. Disponible en: https://actualicese. com/actualidad/2018/02/15/que-tan-beneficioso-resulta-aceptarlos-seguros-de-desempleo-que-ofrecen-las-entidades-bancarias/.

40. Zúñiga Romero M. Despido por necesidades de la empresa y respuesta del mercado asegurador. Paralelo entre la legislación colombiana y la legislación española. Vniversitas [internet]. 2012 [citado 2018 mar. 27]; 61(125) 377-95. Disponible en: http:// www.redalyc.org $/ \mathrm{html} / 825 / 82528730013 /$.

41. Rodríguez Argüello SI. Seguro de desempleo en Colombia, un estudio comparado con España [internet]. Universidad Católica de Colombia [internet]; 2015 [citado 2017 may. 7]. Disponible en: http://repository.ucatolica.edu.co/bitstream/10983/3602/4/ VERSIÓN FINAL SEGURO DE DESEMPLEO.pdf.

42. Cortés González JC. Seguro de desempleo: un compromiso inaplazable de la protección social. En: Herrera Vergara JR et al., editores académicos, El derecho del trabajo y de la seguridad social. Discusiones y debates. Bogotá: Editorial Universidad del Rosario; 2009. pp. 226-44.

43 Mejía Ortega LM, Franco Giraldo A. Protección Social y Modelos de Desarrollo en América Latina. Rev Fac Nac Salud Pública [internet]. 2007 [citado 2019 sep. 8]; 9(3):471-483. Disponible en: http://www.scielo.org.co/pdf/rsap/v9n3/v9n3a16.pdf.

44. Núñez Méndez J, Cuesta L. Propuesta para el diseño de un sistema de protección social para Colombia. Documentos CEDE [internet]. 2006 [citado 2019 jun. 29]. Disponible en: https://issuu.com/ carlosgutierrez30/docs/propuesta_para_el_dise_o_de_un_sis/1.

45. Velásquez Pinto MD. Un análisis de la protección ante el desempleo en América Latina. En: Protección y formación: instituciones para mejorar la inserción laboral en América Latina y Asia. Santiago: CEPAL; 2016. pp. 87-116. 